\title{
Design of broadband power amplifier based on continuous class-F mode with frequency parameterization
}

\author{
Gang Liu ${ }^{1,2}$, Fuqi Mu${ }^{1}$, Xin Qiu ${ }^{1}$, Yongqing Leng ${ }^{1 \mathrm{a})}$, and Xuan Peng ${ }^{1}$
}

\begin{abstract}
This paper investigates the design space of a broadband continuous Class-F power amplifier (PA) at the device package plane. By parameterizing the empirical parameter by frequency, the extrinsic fundamental and second harmonic impedances can be engineered to rotate clockwise on the Smith chart which can be realized by the realistic matching network. The proposed design methodology is verified with the implementation of $10 \mathrm{~W}$ PA which operates across $1.7-3 \mathrm{GHz}$. The experimental results show that this PA can achieve drain efficiency of $54.7 \%-74.8 \%$ in the whole interesting band.

Keywords: broadband, high-efficiency power amplifier, continuous Class-F mode, frequency parameterization, clockwise rotation

Classification: Microwave and millimeter-wave devices, circuits, and modules
\end{abstract}

\section{Introduction}

Broadband and high efficiency are two key requirements in power amplifier (PA) design for forthcoming wireless communication [1]. To improve efficiency of the PA, several kinds of configurations have been widely used, such as Class-E [2, 3, 4], Class-J [5, 6], Class-F/F $\mathrm{F}^{-1}[7,8]$. Nevertheless, Class-E and Class- $\mathrm{F} / \mathrm{F}^{-1}$ need precise harmonic impedance control which is impractical in broadband applicaiton. Class-J amplifier was originally proposed to alleviate this shortcomings. It uses a capacitive harmonic load at the second harmonic rather than a resonant impedance condition. Then, the class-J principle was extended to both the Class-F and inverse Class-F modes in the form of the continuous Class-F [9] and continuous inverse Class-F [10], respectively. To further expand the design space, several hybrid continuous modes and resistive-reactive continuous modes appreared in succession $[11,12,13,14,15,16,17$, $18,19]$. However, the design space of continuous modes mentioned above is derived at the current generate (I-gen) plane while the matching network is constructed at the package plane [20]. Therefore, the theoretical design space can not provide an intuitive solution for actual PA circuit design. Another problem is the anticlockwise rotation of fundamental and second harmonic impedances at package

${ }^{1}$ Institute of Microelectronics, Chinese Academy of Sciences, Beijing 100029, China

${ }^{2}$ University of Chinese Academy of Sciences, Beijing 100049, China

a) lengyongqing@ime.ac.cn

DOI: $10.1587 /$ elex.16.20190038

Received January 14, 2019

Accepted February 19, 2019

Publicized March 8, 2019

Copyedited March 25, 2019 plane with the frequency increases [21, 22]. However, all practical matching networks require the impedances to be rotated in a continuous clockwise fashion on the Smith chart across the frequency band [23].

In this paper, a further investigate into the design space of continuous Class-F mode is carried out. By selecting a frequency parameterization for the continuous Class-F mode, practicable impedances can be provided at the device package plane after projection with the typical package equivalent circuit in [24]. Moreover, a 10-W PA operating in the frequency range of 1.7 to $3 \mathrm{GHz}$ with drain efficiency between $54.7 \%-74.8 \%$ is implemented based on these feasible impedances.

\section{Review of the continuous Class-F mode}

The continuous Class-F mode is a promising solution for high-efficiency and wideband requirements. This mode provides multiple solutions by multiplication of drain voltage waveform of Class-F by the empirical factor $1-\gamma \sin \theta$ as follows:

$$
v_{D S}(\theta)=\left(1-\frac{2}{\sqrt{3}} \cos \theta+\frac{1}{3 \sqrt{3}} \cos 3 \theta\right) \cdot(1-\gamma \sin \theta)
$$

For $\gamma=0$, the standard Class-F voltage waveform is obtained with theoretical drain efficiency (DE) of $90.7 \%$. Each value of $\gamma$ represents particular loads at I-gen plane of device represented as [25]

$$
\begin{aligned}
& Z_{1 \text { int }}=\left(\frac{2}{\sqrt{3}}-j \cdot \gamma\right) \cdot R_{o p t} \\
& Z_{2 \text { int }}=j \frac{7 \sqrt{3} \pi}{24} \gamma \cdot R_{\text {opt }}
\end{aligned}
$$

where $Z_{1 \text { int }}$ and $Z_{2 \text { int }}$ are the fundamental and second harmonic impedances, respectively. And the open circuit is presented to the third harmonic. $R_{\text {opt }}$ is the optimal fundamental impedance of standard Class-B [26]. Each pair of $Z_{1 \text { int }}$ and $Z_{2 i n t}$ delivers the same output power and efficiency as standard Class-F.

\section{Design space analysis at package plane}

In practice, the impedance at package plane is preferred for actual PA circuit. By employing the typical package equivalent circuit, the theoretical design space can be transformed to the package plane as shown in Fig. 1. $Z_{1 p k g}$ and $Z_{2 p k g}$ are the fundamental and second harmonic impedances at the package plane, respectively. It can be seen 


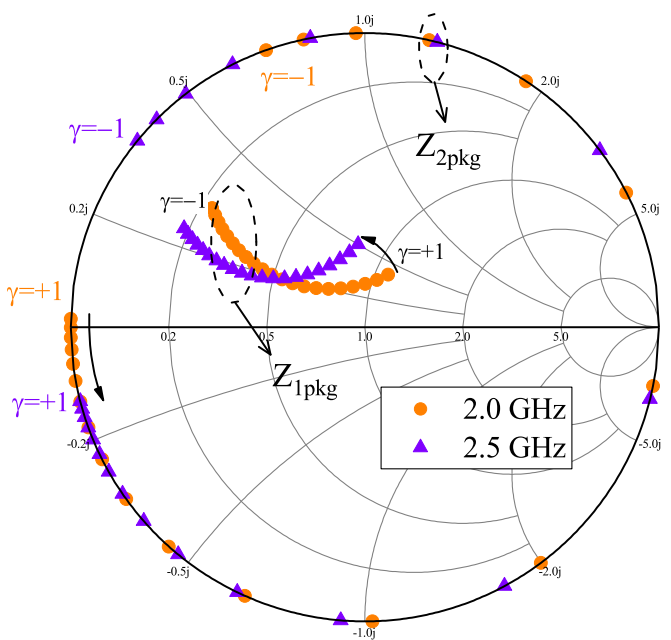

Fig. 1. The optimal impedances at package plane with different frequencies.

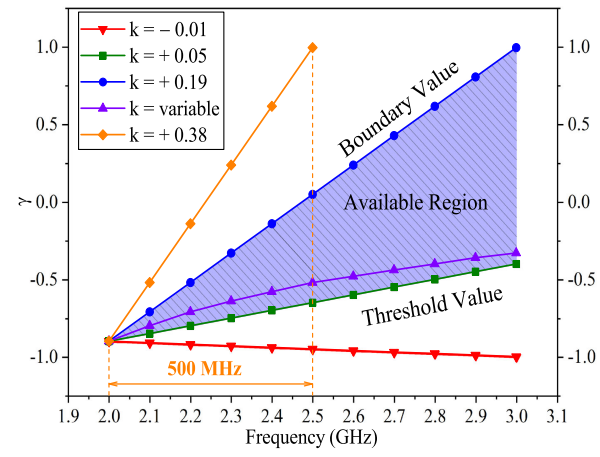

(a)

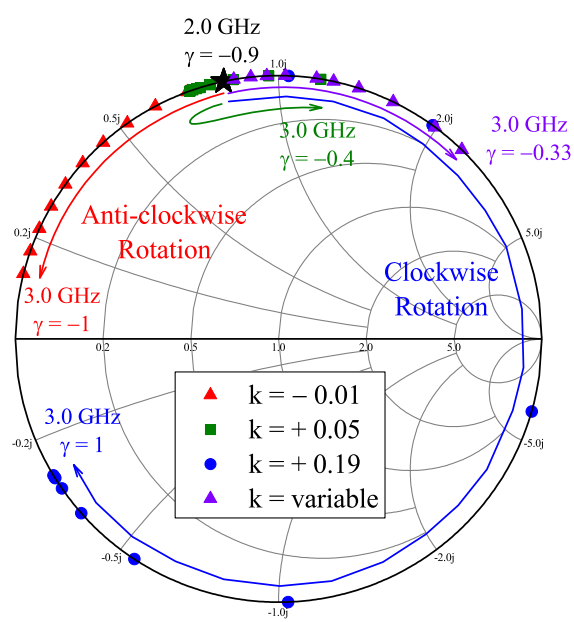

(b)

Fig. 2. (a) Different $k$ for the same starting value of $\gamma=-0.9$ and (b) their corresponding $Z_{2 p k g}$.

that the rotation of fundamental and second harmonic impedances is anticlockwise as the frequency increases, especially the extent of second harmonic impedance is widely distributed. Therefore, it is impossible to be realized through the passive matching network. To overcome this problem, it is necessary to explore the design space by analyzing the parameter $\gamma$. There are three considerasions: gradient of $\gamma$, range of $\gamma$ and bandwidth of operating band.

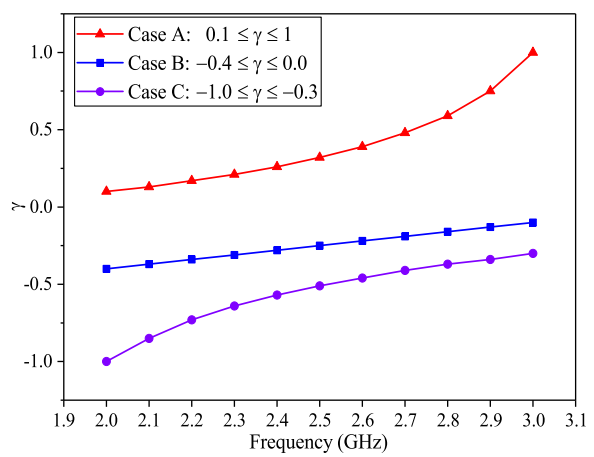

(a)

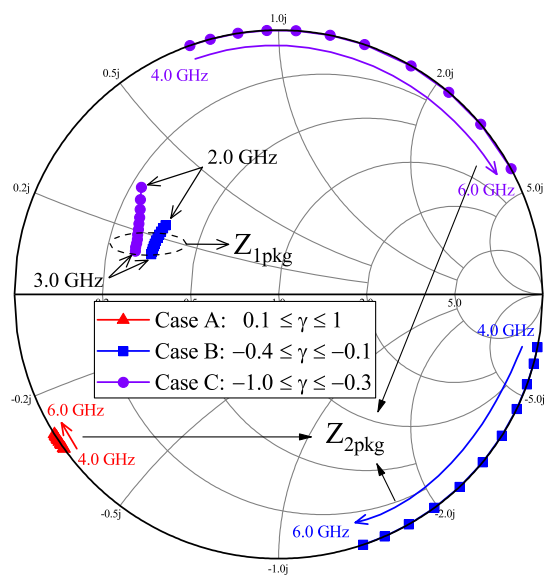

(b)

Fig. 3. (a) Different ranges of $\gamma$ and (b) their corresponding $Z_{1 p k g}$ and $Z_{2 p k g}$.

Before analysis, $k$, the gradient of $\gamma$, is defined as the difference between successive values of $\gamma$ for every successive change in frequency. Three different cases with different $k$ have been shown in Fig. 2(a) and their corresponding $Z_{2 p k g}$ on the Smith chart in Fig. 2(b). In all cases, $\Delta f$ is set to be $100 \mathrm{MHz}$ to analysis the effect of $k$. And $\gamma$ is set to -0.9 at $2 \mathrm{GHz}$ for all cases. It can be seen that $k$ can be positive or negative. A negative $k$ makes the $Z_{2 p k g}$ follow an anticlockwise rotation as shown in Fig. 2(b). However, for $k=0.05$, the extent of anticlockwise rotation reduces greatly and a clockwise rotation begins. This value is defined as threshold value. Considering the boundary of $\gamma$, there will be a boundary value of $k$, which is shown in Fig. 2(a). Any further increase in $k$ will make the bandwidth decrease. For $k=0.38$, only $500 \mathrm{MHz}$ of band can be obtained. As discussed above, the constant value of $k$ does not provide an good solution for the implementation of the matching network. Thus, a variable $k$ is being investigated and the resultant values of $Z_{2 p k g}$ are shown in Fig. 2(b). Obviously, a variable $k$ can provide a more feasible solution. Therefore, the impedance trajectory can be customized by changing the value of $k$ at every frequency point to meet specific demand. It shoule be noted that the values of $k$ are different for different range of $\gamma$.

In order to analysis range of the values of $\gamma$, three different cases are shown in Fig. 3(a); case A is $-1 \leq \gamma \leq$ -0.5 , case $\mathrm{B}$ is $-0.4 \leq \gamma \leq 0$, and case $\mathrm{C}$ is $0.1 \leq \gamma \leq 1$. 
And the corresponding values of $Z_{2 p k g}$ are shown in Fig. 3(b). It can be seen that all cases can lead to clockwise rotation. For case A, however, the extent of clockwise rotation is very small, which is impossible to realize in wideband applicaiton. Therefore, the suitable range of $\gamma$ is -1 to 0 . Although case $B$ and case $C$ have similar extent of rotation of the second harmonic impedances, case $\mathrm{C}$ is more suitable for the implementation of a passive network when considering the fundamental impedance matching.

\section{PA design and measurement}

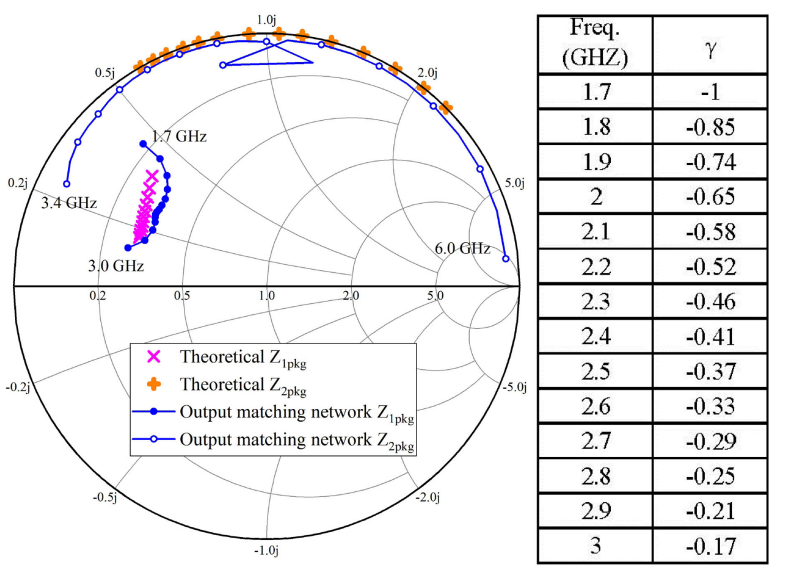

(a)

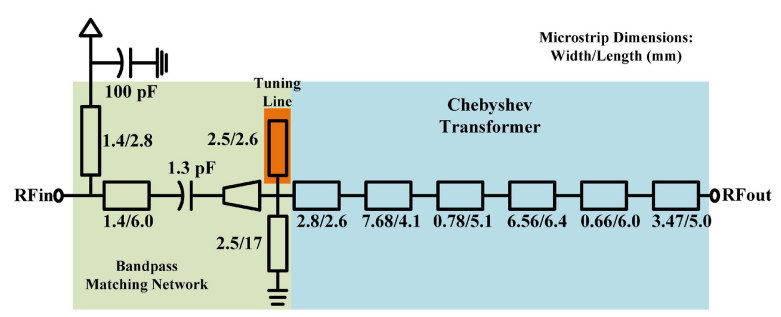

(b)

Fig. 4. (a) The theoretical impedances at package plane and the output matching network impedance trajectories (b) The output matching network with the dimensions.

The 10W Cree CGH40010F device is used in this design to verify the proposed theory. The drain voltage is set at $28 \mathrm{~V}$ with a quiescent current of $106 \mathrm{~mA}$. And a parallel circuit consisting of $47 \Omega$ and $3.6 \mathrm{pF}$ is employed to ensure the stability.

Based on the previous analysis, $-1 \leq \gamma \leq-0.17$ is selected and $k$ is set as a variable. The value of $k$ of each frequency and the resultant $Z_{1 p k g}$ and $Z_{2 p k g}$ are shown in Fig. 4(a). It can be seen that the fundamental loads are slightly to follow a clockwise rotation besides the clockwise rotation of the second harmonic loads. Therefore, these loads can be realized by passive matching network. The output matching network is composed of bandpass matching network [27] and step-impedance filtering transformer [28, 29]. And the tuning line is added to adjust the second harmonic impedances. It can be seen that the fundamental and the second harmonic impedances basically satisfy the theoretical impedance requirement, although some mismatches exist. Considering the nonlinear

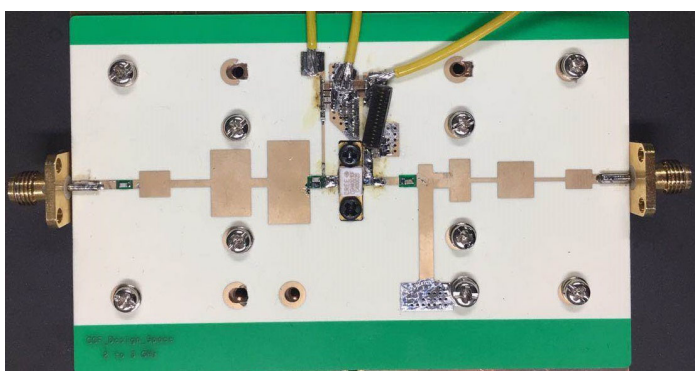

Fig. 5. Photograph of fabricated circuit.

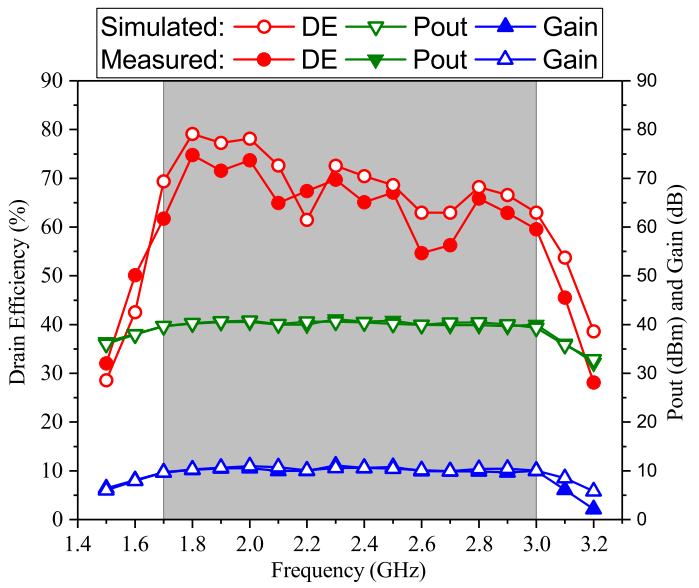

Fig. 6. Measured and simulated DE, output power, and gain.

characteristic of the drain-source capacitor, these mismatches are acceptable [30].

The circuit is realized on Roger's 4350B substrate with 3.66 as dielectric constant and 30 mil of height. Fig. 5 illustrates the photograph of the fabricated circuit. A $3.5 \mu \mathrm{H} \mathrm{4310LC} \mathrm{ferrite} \mathrm{is} \mathrm{used} \mathrm{to} \mathrm{supply} \mathrm{very} \mathrm{nice} \mathrm{bias}$ performance. Fig. 6 shows the measured and simulated large-signal test results over the whole bandwidth using an input power of $30 \mathrm{dBm}$. The simulated DE varies between $61.5 \%$ and $79.1 \%$ whereas the measured DE varies between $54.7 \%$ and $74.8 \%$ over the operating band from 1.7 to $3.0 \mathrm{GHz}$. Output power varies between $39.3-$ $40.8 \mathrm{dBm}$ and $39.7-41.1 \mathrm{dBm}$ in simulation and measurement, respectively. The gain variation is between 9.7-11 $\mathrm{dB}$ in both simulation and measurement. The comparison with the recently reported broadband PA results is presented in Table I.

Table I. Comparison of some published PAs and our work

\begin{tabular}{|c|c|c|c|c|}
\hline Ref & $\begin{array}{c}\text { Bandwidth } \\
(\mathrm{GHz})\end{array}$ & $\begin{array}{c}\text { Power } \\
(\mathrm{dBm})\end{array}$ & $\begin{array}{c}\mathrm{DE} \\
(\%)\end{array}$ & $\begin{array}{c}\text { Gain } \\
(\mathrm{dB})\end{array}$ \\
\hline $2016[12]$ & $2.85-4.25$ & $40.7-42.0$ & $58-78$ & $10.8-12$ \\
\hline $2016[13]$ & $2.4-3.9$ & $39.6-41.4$ & $62.2-74.7$ & $10.7-12.5$ \\
\hline $2017[17]$ & $2.5-4.1$ & $39.6-42.6$ & $47-75$ & $8-14$ \\
\hline $2017[18]$ & $2.0-3.0$ & $39.5-41.6$ & $58-72$ & $11.5-12.5$ \\
\hline This work & $1.7-3.0$ & $39.7-41.1$ & $54.7-74.8$ & $9.7-11$ \\
\hline
\end{tabular}




\section{Conclusion}

This paper analyzes the design space of continuous Class-F mode at the package plane by using the package model. By parameterizing the empirical parameter $\gamma$ by frequency, the impedance trajectories of fundamental and second harmonic can be modified to be more realizable for matching circuit. Based on this analysis, a highly efficient broadband PA has been fabricated displaying good performance.

\section{Acknowledgments}

This work was supported by the National Natural Science Foundation of China under Grant 61501455 and Natural Science Foundation of Beijing Municipality under Grant 4162068 .

\section{References}

[1] S. C. Cripps: RF Power Amplifiers for Wireless Communications (Artech House, Norwood, MA, USA, 1999).

[2] N. Sokal and A. Sokal: "Class E - A new class of high-efficiency tuned single-ended switching power amplifiers," IEEE J. SolidState Circuits 10 (1975) 168 (DOI: 10.1109/JSSC.1975.1050582).

[3] C. Rong, et al.: "A class E GaN microwave power amplifier accounting for parasitic inductance of transistor," IEEE Electron. Express 14 (2017) 20170127 (DOI: 10.1587/elex.14.20170127).

[4] J. Zhou, et al.: "Improved reactance-compensation technique for the design of wideband suboptimum class-E power amplifiers," IEEE Trans. Microw. Theory Techn. 63 (2015) 2793 (DOI: 10.1109/TMTT.2015.2455505)

[5] Z. Hu, et al.: "C-band general class-J power amplifier using GaN HEMT," IEICE Electron. Express 13 (2016) 20160483 (DOI: 10.1587/elex.13.20160483).

[6] A. Alizadeh and A. Medi: "A broadband integrated class-J power amplifier in GaAs pHEMT technology," IEEE Trans. Microw. Theory Techn. 64 (2016) 1822 (DOI: 10.1109/TMTT.2016. 2552167).

[7] F. H. Raab: "Class-F power amplifiers with maximally flat waveforms," IEEE Trans. Microw. Theory Techn. 45 (1997) 2007 (DOI: 10.1109/22.644215).

[8] Y. Ding, et al.: "High-efficiency concurrent dual-band class-F and inverse class-F power amplifier," Electron. Lett. 47 (2011) 847 (DOI: 10.1049/el.2011.1662).

[9] N. Tuffy, et al:: "A simplified broadband design methodology for linearized high-efficiency continuous class-F power amplifiers," IEEE Trans. Microw. Theory Techn. 60 (2012) 1952 (DOI: 10. 1109/TMTT.2012.2187534).

[10] M. Yang, et al:: "Highly efficient broadband continuous inverse class-F power amplifier design using modified elliptic low-pass filtering matching network," IEEE Trans. Microw. Theory Techn. 64 (2016) 1515 (DOI: 10.1109/TMTT.2016.2544318).

[11] P. Wright, et al.: "A methodology for realizing high efficiency class-J in a linear broadband PA," IEEE Trans. Microw. Theory Techn. 57 (2009) 3196 (DOI: 10.1109/TMTT.2009.2033295).

[12] K. Chen and D. Peroulis: "Design of broadband highly efficient harmonic-tuned power amplifier using in-band continuous class$\mathrm{F}^{-1} / \mathrm{F}$ mode transferring," IEEE Trans. Microw. Theory Techn. 60 (2012) 4107 (DOI: 10.1109/TMTT.2012.2221142).

[13] W. Shi, et al.: "A series of inverse continuous modes for designing broadband power amplifiers," IEEE Microw. Wireless Compon. Lett. 26 (2016) 525 (DOI: 10.1109/LMWC.2016.2574820).

[14] C. Friesicke, et al.: "The resistive-reactive class-J power amplifier mode," IEEE Microw. Wireless Compon. Lett. 25 (2015) 666 (DOI: 10.1109/LMWC.2015.2463211).

[15] Q. Li, et al.: "Extend the class-B to class-J continuum mode by adding arbitrary harmonic voltage elements," IEEE Microw. Wireless Compon. Lett. 26 (2016) 522 (DOI: 10.1109/LMWC.
2016.2574824).

[16] W. Shi, et al.: "A series of inverse continuous modes for designing broadband power amplifiers," IEEE Microw. Wireless Compon. Lett. 26 (2016) 525 (DOI: 10.1109/LMWC.2016.2574820).

[17] M. Zhang, et al.: "Design of broadband inverse class-F based on resistive-reactive series of inverse continuous modes," IEICE Electron. Express 14 (2017) 20170537 (DOI: 10.1587/elex.14. 20170537).

[18] X. Meng, et al.: "Design approach for implementation of class-J broadband power amplifiers using synthesized band-pass and lowpass matching topology," IEEE Trans. Microw. Theory Techn. 65 (2017) 4984 (DOI: 10.1109/TMTT.2017.2711021).

[19] C. Huang, et al:: "Design of broadband high-efficiency power amplifiers based on the hybrid continuous modes with phase shift parameter," IEEE Microw. Wireless Compon. Lett. 28 (2018) 159 (DOI: 10.1109/LMWC.2017.2787061).

[20] J. Chen and S. He: "Broadband high-efficiency power amplifiers design based on hybrid continuous modes utilizing the optimal impedances at package plane," IEEE MTT-S Int. Microw. Symp. (2015) 1 (DOI: 10.1109/MWSYM.2015.7166728).

[21] W. Demenitroux, et al:: "Multiharmonic volterra model dedicated to the design of wideband and highly efficient GaN power amplifiers," IEEE Trans. Microw. Theory Techn. 60 (2012) 1817 (DOI: 10.1109/TMTT.2012.2191305).

[22] D. Y.-T. Wu, et al.: "Design of a broadband and highly efficient $45 \mathrm{~W}$ GaN power amplifier via simplified real frequency technique," IEEE MTT-S Int. Microw. Symp. (2010) 1090 (DOI: 10.1109/MWSYM.2010.5517636).

[23] T. Sharma, et al.: "A methodology for implementation of highefficiency broadband power amplifiers with second-harmonic manipulation," IEEE Trans. Circuits Syst. II, Exp. Briefs 63 (2016) 54 (DOI: 10.1109/TCSII.2015.2482139).

[24] P. J. Tasker and J. Benedikt: "Waveform inspired models and the harmonic balance emulator," IEEE Microw. Mag. 12 (2011) 38 (DOI: 10.1109/MMM.2010.940101).

[25] V. Carrubba, et al:: "On the extension of the continuous class-F mode power amplifier," IEEE Trans. Microw. Theory Techn. 59 (2011) 1294 (DOI: 10.1109/TMTT.2011.2117435).

[26] A. Grebennikov and N. O. Sokal: Switchmode RF and Microwave Power Amplifiers (Newnes, Burlington, MA, New York, 2007).

[27] P. Saad, et al:: "Design of a highly efficient 2-4-GHz octave bandwidth GaN-HEMT power amplifier," IEEE Trans. Microw. Theory Techn. 58 (2010) 1677 (DOI: 10.1109/TMTT.2010. 2049770).

[28] J. Xia, et al.: "A linearized $2-3.5 \mathrm{GHz}$ highly efficient harmonictuned power amplifier exploiting stepped-impedance filtering matching network," IEEE Microw. Wireless Compon. Lett. 24 (2014) 602 (DOI: 10.1109/LMWC.2014.2324752).

[29] G. L. Matthaei: "Tables of chebyshev impedance-transforming networks of low-pass filter form," Proc. IEEE 52 (1964) 939 (DOI: 10.1109/PROC.1964.3185).

[30] J. Moon, et al.: "Investigation of a class-J power amplifier with a nonlinear $\mathrm{C}_{\text {out }}$ for optimized operation," IEEE Trans. Microw. Theory Techn. 58 (2010) 2800 (DOI: 10.1109/TMTT.2010. 2077970). 